\title{
10-year trends in the incidence of microtia: a nationwide population-based study from Taiwan
}

\author{
Yen-Fu Cheng ${ }^{1,2,3,4} \cdot$ Po-Ting $\operatorname{Lin}^{5} \cdot$ Herng-Ching $\operatorname{Lin}^{6}(\mathbb{0}$
}

Received: 8 August 2021 / Accepted: 10 August 2021 / Published online: 19 August 2021

(c) The Author(s), under exclusive licence to Springer-Verlag GmbH Germany, part of Springer Nature 2021

\section{To the Editor,}

We would like to thank the editor for the opportunity to respond to the issues raised in Drs. Guo and Zhang's letter. We also thank Drs. Guo and Zhang for their interest in our paper [1] and for taking the time to express their opinions.

In their letter to the editor, Drs. Guo and Zhang were concerned about an underestimated prevalence of microtia/anotia due to the lack of inclusion of stillbirth/termination data. They also suggested exploration of the etiology of microtia/ anotia in the study. While we welcome these comments and suggestions, we do want to clarify some points in response to their concerns.

The main objective of this study, as suggested by the title and context of our paper, was to analyze the 10-year secular trends in the incidence of microtia and/or anotia in Taiwan. We were very direct and clear throughout our article that we retrieved data of children aged $\leq 1$ year using Taiwan's nationwide population-based data (National Health Insurance Research Dataset, NHIRD), which is based on medical

This reply refers to the comment available online at https://doi.org/ 10.1007/s00405-021-07032-9.

\section{Herng-Ching Lin}

henry11111@tmu.edu.tw

1 Department of Medical Research, Taipei Veterans General Hospital, Taipei, Taiwan

2 Department of Otolaryngology-Head and Neck Surgery, Taipei Veterans General Hospital, Taipei, Taiwan

3 Department of Otolaryngology-Head and Neck Surgery, School of Medicine, National Yang-Ming University, Taipei, Taiwan

4 Research Center of Sleep Medicine, College of Medicine, Taipei Medical University, Taipei, Taiwan

5 Weinberg College of Arts and Sciences, Northwestern University, Evanston, IL, USA

6 School of Health Care Administration, College of Management, Taipei Medical University, Taipei, Taiwan care claims records of the single-payer National Health Insurance Program of Taiwan. Therefore, data such as stillbirths, terminations of pregnancy, and fetuses $<28$ weeks of gestation are not available. In fact, all reported incidence and prevalence of microtia do not come along with data of stillbirth/termination. Even with the addition of these data, we believe that the 10-year trend in the incidence of microtia/anotia should not be affected.

While Drs. Guo and Zhang expressed the necessity of exploring etiology and genetic inheritance of microtia/ anotia to enhance the persuasiveness of our study, we want to emphasize that these suggestions are out of the scope of this paper. As mentioned in the study limitation, many patient characteristics such as genetic and laboratory data are not available in Taiwan's NHIRD. Nevertheless, this paper solely focused on the trend on incidence of microtia. While it may be insightful for other studies to investigate the etiology of microtia/anotia and its association with other syndromic diseases in the future, further investigation in this field is ultimately beyond the framework of this particular study of ours.

We do, however, appreciate the finding of the reversed order of Figs. 2 and 3. We have requested the editorial correction before the publication of this paper.

\section{Reference}

1. Cheng YF, Xirasagar S, Liu TC, Kuo NW, Lin HC (2021) Tenyear trends in the incidence of microtia: a nationwide populationbased study from Taiwan. Eur Arch Otorhinolaryngol. Online ahead of print

Publisher's Note Springer Nature remains neutral with regard to jurisdictional claims in published maps and institutional affiliations. 